\title{
Adenocarcinoma de próstata metastático em paciente com 50 anos: estudo de caso
}

\author{
Metastatic prostate adenocarcinoma in a 50-year-old patient: case study
}

Adenocarcinoma de próstata metastático en un paciente de 50 años: estudio de caso

Marcelle da Silva Costa ${ }^{1 *}$, Jheniffer Bastos da Mata ${ }^{1}$, Soraya Aires Dias ${ }^{1}$, Pedro Manuel Gonzalez Cuellar ${ }^{1}$.

\section{RESUMO}

Objetivo: Investigar os fatores clínico-patológicos e prognósticos de pacientes com adenocarcinoma de próstata avançado com idade $<55$ anos. Detalhamento do caso: Homem, 51 anos, apresenta um histórico de dor e dificuldade para urinar há aproximadamente dois anos, sendo tratado apenas sintomaticamente. Em junho de 2020 manifestou piora do quadro urológico e além do estado prévio, apresentava dores intensas em membros inferiores, associado a dificuldade de deambular. Foi solicitado Antígeno Prostático Específico Total (PSA), constatando um valor de $2.875,76 \mathrm{ng} / \mathrm{ml}$, realizando em seguida Ressonância Magnética da próstata e coluna lombar, cintilografia óssea e biópsia transretal prostática, cujo último constatou Adenocarcinoma Acinar e usual com Grau de Gleason 9. Considerações finais: Ainda que não haja consenso científico a respeito de quando se deve dar início a triagem para câncer prostático, é irrefutável a importância de uma abordagem individual voltada para suspeita de neoplasia prostática frente a sintomas urinários persistentes, uma vez que, diagnóstico precoce favorece um prognóstico mais favorável em pacientes jovens.

Palavras-chave: Neoplasia de próstata, Escore de Gleason, PSA.

\begin{abstract}
Objective: To investigate the clinical-pathological and prognostic factors of patients with advanced prostate cancer at the age under 55 years old. Details of the case: A 51-year-old man has a history of pain and difficulty urinating for approximately two years, being treated only symptomatically. In June 2020, the urological condition worsened and, in addition to his previous condition, he presented severe pain in the lower limbs associated with difficulty walking. Specific Prostatic Antigen (PSA) was requested, with a value of 2,875.76 $\mathrm{ng} / \mathrm{ml}$ then were performed Magnetic resonance imaging of the prostate and lumbar spine, bone scintigraphy and prostatic transrectal biopsy, the latter found Acinar and usual Adenocarcinoma with Gleason Grade 9. Final considerations: Although there is no scientific consensus as to when screening for prostate cancer should begin, the importance of an individual approach towards suspected prostatic carcinoma in cases of persistent urinary symptoms is irrefutable, since early diagnosis favors a more favorable prognosis in young patients.
\end{abstract}

Key words: Prostate cancer, Gleason grading, PSA.

\section{RESUMEN}

Objetivo: Investigar los factores clínico-patológicos y pronósticos de pacientes $<55$ años con adenocarcinoma de próstata avanzado. Detalles del caso: Un hombre de 51 años tiene antecedentes de dolor y dificultad para orinar durante aproximadamente dos años, siendo tratado solo sintomáticamente. En junio de 2020 su estado

${ }^{1}$ Universidade Federal do Tocantins (UFT), Palmas - TO. *E-mail: marcelle.s.costa@mail.uft.edu.br 
urológico empeoró y, además de su estado anterior, presentaba fuertes dolores en miembros inferiores, asociados a dificultad para caminar. Se solicitó Antígeno Prostático Específico Total (PSA), encontrándose un valor de 2.875,76 $\mathrm{ng} / \mathrm{ml}$, luego se realizó Resonancia Magnética de próstata y columna lumbar, gammagrafía ósea y biopsia transectal de próstata, en esta última se encontró Acinar y Adenocarcinoma habitual con Grado de Gleason 9. Consideraciones finales: aunque no existe un consenso científico sobre cuándo debe comenzar el cribado del cáncer de próstata, la importancia de un enfoque individual dirigido a la sospecha de cáncer de próstata frente a los síntomas urinarios persistentes es irrefutable, dado que el diagnóstico precoz favorece un pronóstico más favorable en pacientes jóvenes.

Palabras clave: Cáncer próstata, Classificacion de Gleason, PSA.

\section{INTRODUÇÃO}

De acordo com o Instituto Nacional do Câncer (INCA), o câncer de próstata é o segundo tipo de câncer de maior incidência em homens, e também, o segundo mais comum considerando ambos os sexos. No Brasil, em 2020, estimam-se 65.840 novos casos desse tipo de tumor, com a segunda maior taxa de mortalidade, ficando atrás apenas das neoplasias que afetam brônquios e pulmões (INCA, 2020).

A neoplasia prostática é considerada um câncer da terceira idade, sendo a idade um dos fatores de risco para o desenvolvimento desse tipo de malignidade. Além da faixa etária, hereditariedade (pai ou irmão com câncer de próstata antes dos 60 anos), origem étnica, excesso de gordura corporal e exposição a aminas aromáticas, aumentam as condições de desenvolver tumor prostático (CZORNY RCN, et al., 2017).

A maioria dos diagnósticos de neoplasia prostática está associada a homens acima de 65 anos, e cerca de $1 \%$, a neoplasia precoce em homens com idades abaixo dos 55 anos. A literatura atual é inconsistente em se tratando do prognóstico de câncer de próstata em adultos jovens. Alguns estudiosos sugeriram que a idade jovem é um indicador de mau prognóstico, outros relatam maior sobrevida nessa faixa etária. Há ainda, estudos que mostram não haver diferença significativa na ocorrência ou grau de estágio da doença em relação a idade (HUANG T-H, et al., 2016).

O adenocarcinoma acinar usual é o tipo histológico mais comum da neoplasia prostática. Ele é avaliado por possuir um bom prognóstico quando diagnosticado e tratado precocemente. A Sociedade Brasileira de Urologia preconiza que a partir dos 50 anos, os homens devem procurar o serviço de saúde para uma avaliação individualizada tendo como objetivo o diagnóstico precoce do câncer de próstata (VAZ DWN, et al., 2020).

Além da morfologia acinar usual que está presente em mais de $90 \%$ dos adenocarcinomas, há dois grupos histológicos que caracterizam os subtipos de câncer de próstata: as variantes do câncer acinar usual e, cânceres com padrão histológico incomuns para a próstata. Das variantes do câncer de próstata usual se encontram os subtipos como pseudo-hiperplásico, glândula espumosa e alterações semelhantes às células de Paneth. Já os tipos incomuns se encontram carcinoma ductal, carcinoma mucinoso, carcinoma de células pequenas, carcinoma sarcomatoide e carcinoma urotelial primário da próstata (MIKUZ G, 2015).

O diagnóstico inicial da neoplasia prostática é baseado em achados clínicos do toque retal e/ou antígeno prostático específico (PSA). A confirmação da neoplasia é feita através da realização do estudo histopatológico do tecido obtido pela biópsia prostática. Através da biópsia é possível determinar o escore de Gleason, que prevê o grau de variação celular e prognóstico da doença (QUIJADA PDS, et al., 2017).

O PSA é um marcador tumoral utilizado no rastreio da neoplasia prostática com repercussão na morbidade e mortalidade. Recentemente são considerados alterados os valores de PSA acima de $2,5 \mathrm{ng} / \mathrm{ml}$. Esse marcador possui alta sensibilidade e baixa especificidade, pois outras causas como prostatite e a hiperplasia prostática podem elevar os valores além de causas tumorais (ARAUJO RM, et al., 2017).

A classificação por Escore de Gleason é uma análise anatomopatológica tecidual da próstata obtida por cirurgia ou biópsia. $O$ escore é estabelecido por um sistema de pontos, que vão de 1 a 5 , para caracterizar o 
aspecto microscópico celular. O resultado final da contagem demonstra o total da pontuação dos dois tipos citológicos tumorais mais frequente no fragmento examinada. De acordo com essa técnica, 1 ponto é atribuído quando as células são mais diferenciadas (análogo ao tecido prostático fisiológico), e 5 quando menos semelhantes, ou seja, mais invasivo. A pontuação de 2 a 4 é usada para quadros interpostos, no intuito do menor para maior agressividade (ARAUJO RM, et al., 2017).

Além da nota, o escore de Gleason é classificado em 5 grupos de acordo com sua pontuação. Biópsias que apresentam escore de Gleason 3+3 é inserido no grupo 1, Gleason 3+4 (grupo 2), Gleason 4+3 (grupo 3), Gleason 4+4, 3+5, 5+3 (grupo 4) e Gleason 9-10 (grupo 5). Esse sistema ofereceu a maior discriminação de prognóstico e tratamento (LITWIN MS e TAN H-J, 2017).

Nos estádios iniciais, a neoplasia prostática é assintomática. Entretanto, a doença pode evoluir com dificuldade miccional, hematúria, dor a micção, hesitação e frequência urinária aumentada, não sendo estes sintomas específicos para a doença (MERRIEL SWD, et al., 2018).

Este relato de caso visa demonstrar como o diagnóstico precoce do câncer tem impacto no prognóstico da doença. Além de levantar a associação de câncer de próstata avançado em relação a idade e seu mau prognóstico.

\section{DETALHAMENTO DO CASO}

O caso a seguir foi autorizado pelo paciente para ser exposto, sendo assinado um Termo de Consentimento Livre Esclarecido (TCLE), constando que seu relato é para fins científicos e que não será divulgado nenhum dado pessoal ou qualquer tipo de identificação do mesmo.

Homem, 51 anos, solteiro, pardo, trabalhador braçal, residente em Dois Irmãos do Tocantins. Apresentouse a assistência básica de saúde municipal no mês de Setembro/2020 com queixa de dor lombar irradiada para membros inferiores há 3 meses, disúria e polaciúria com início há 2 anos.

Paciente relata que aproximadamente há dois anos atrás vem sentindo quadros de disúria e polaciúria com frequência, sendo tratado com antibioticoterapia para infecções do trato urinário e analgesia. Conta que o jato urinário reduziu e a coloração da urina está mais escura desde essa época (sugerindo hematúria).

Além das queixas urinárias, descreve que vem sentindo uma dor intensa, contínua, insidiosa, persistente e sem fatores de melhora ou piora na região lombo-sacra, pelve e perna direita. Associada a dor refere dificuldade de deambular e adinamia. Nega história familiar para neoplasias ou outras comorbidades.

Ao exame físico, o paciente encontrava-se em bom estado geral, sem alterações dos sinais vitais, abdome flácido, indolor, com massa palpável em fossa ilíaca direita, esquerda e hipogástrio. Não foi realizado toque retal na primeira consulta.

Foi iniciado investigação complementar com Ressonância Nuclear Magnética (RNM) da coluna lombossacra e dosagem de PSA total e livre. A RNM evidenciou múltiplas lesões de aspecto expansivo nos corpos vertebrais, sacro e asas ilíacas; fratura patológica em corpo de L2 e lesão de processo expansivo dentro da bexiga associado a linfonodomegalia inguinal (Figura 1). Já o PSA total foi de $2875,76 \mathrm{ng} / \mathrm{ml}$ e o livre de $25 \mathrm{ng} / \mathrm{ml}$.

Diante de tais resultados prosseguiu-se a investigação e estadiamento com Ressonância Magnética Multiparamétrica da Próstata (RMMP), cintilografia e biópsia transretal prostática. A RMMP evidenciou uma próstata de contornos lobulares com cápsula irregular sugerindo extensão extraprostática, em corte coronal e sagital mostrou próstata com hipossinais em T2 e bexiga com paredes espessadas e conteúdo espesso (Figura 2, Figura 3 e Figura 4). Isso indica uma formação de caráter expansiva/infiltrativa em quase totalidade da próstata, incluindo componente prostático intravesical e bexiga urinária com paredes trabeculadas corroborando com a ideia de bexiga de esforço. 
Figura 1 - Ressonância Nuclear Magnética de Coluna Lombossacra com lesões de aspecto expansivo nos elementos ósseos.

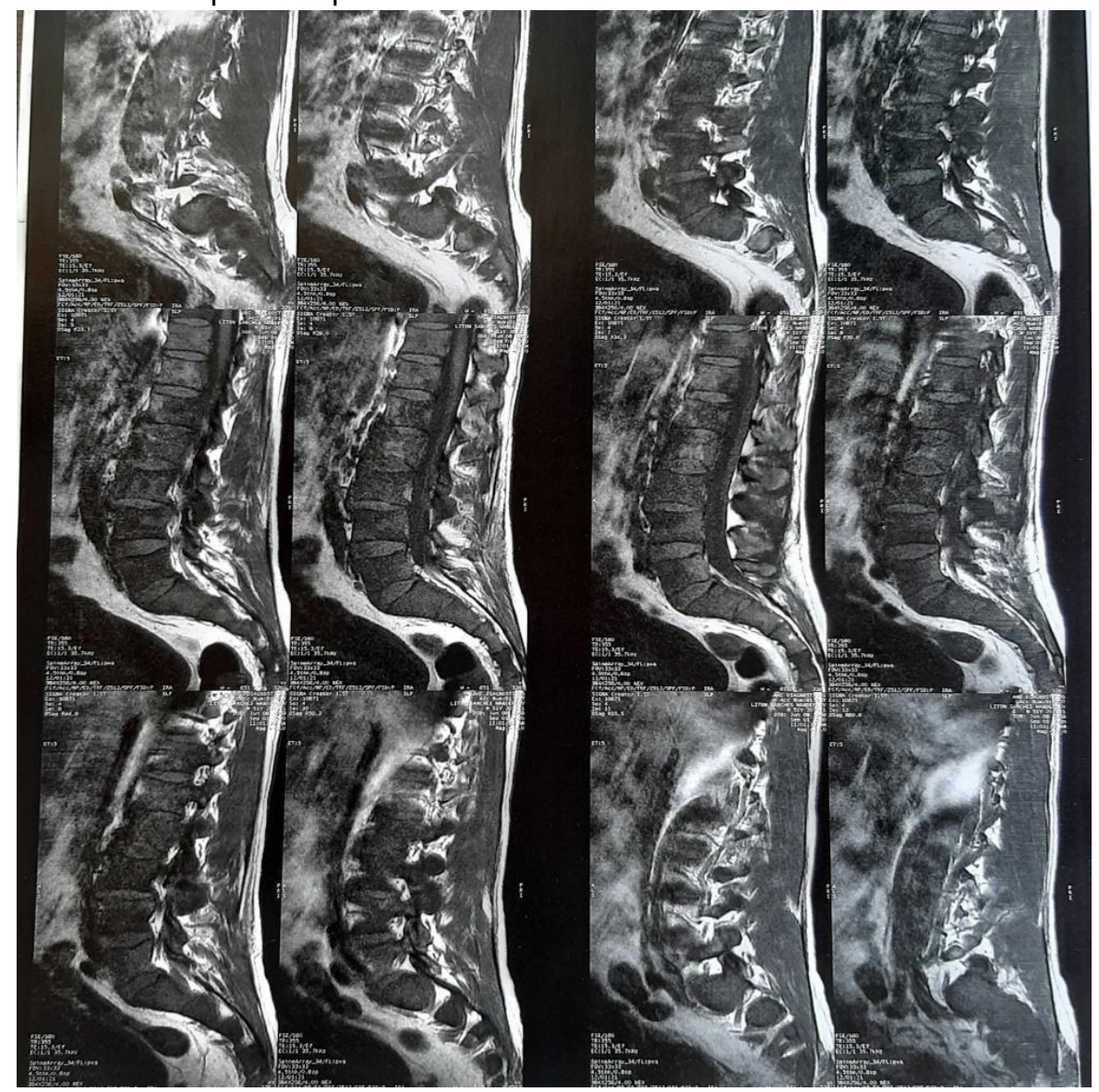

Fonte: Costa MS, et al., 2021.

Figura 2 - Ressonância Multiparamétrica da próstata em corte axial.

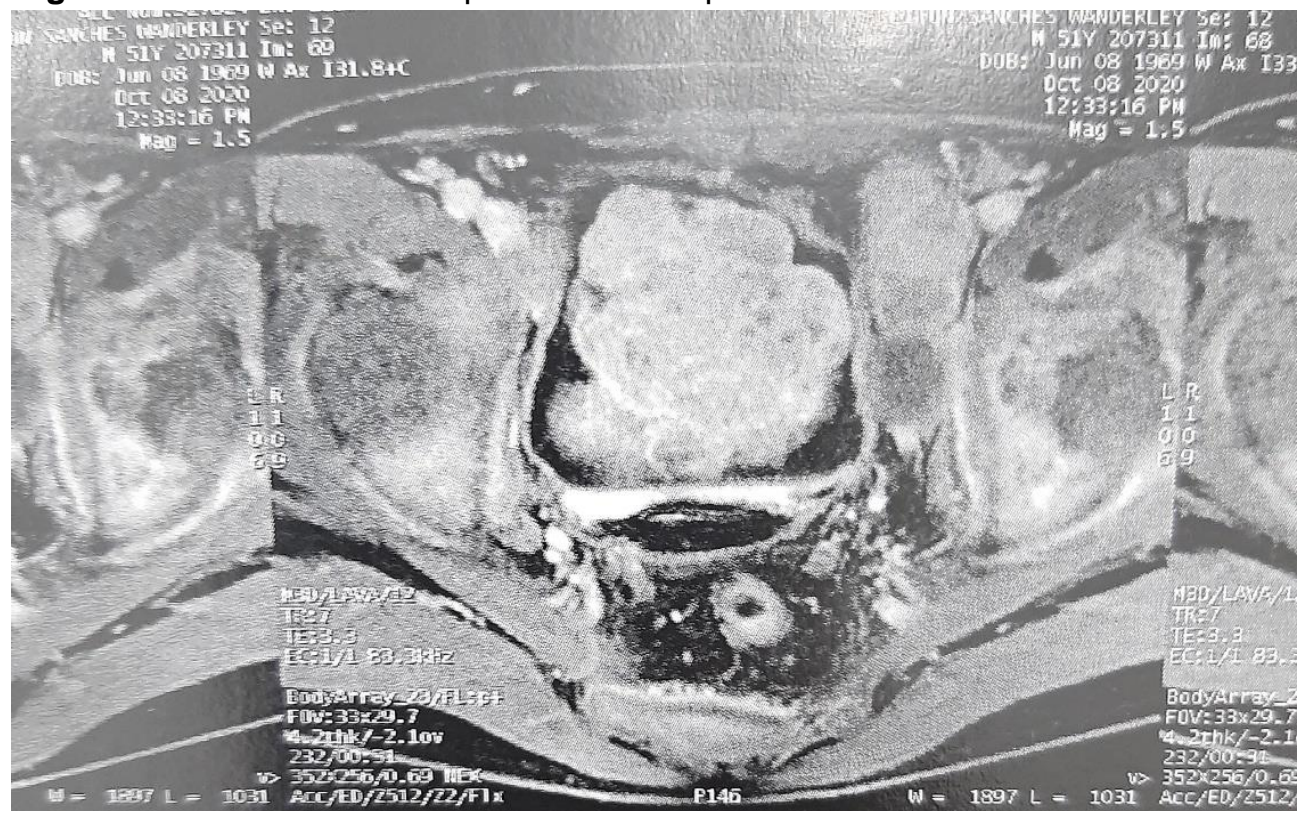

Fonte: Costa MS, et al., 2021. 
Figura 3 - Ressonância Multiparamétrica da próstata em corte coronal.

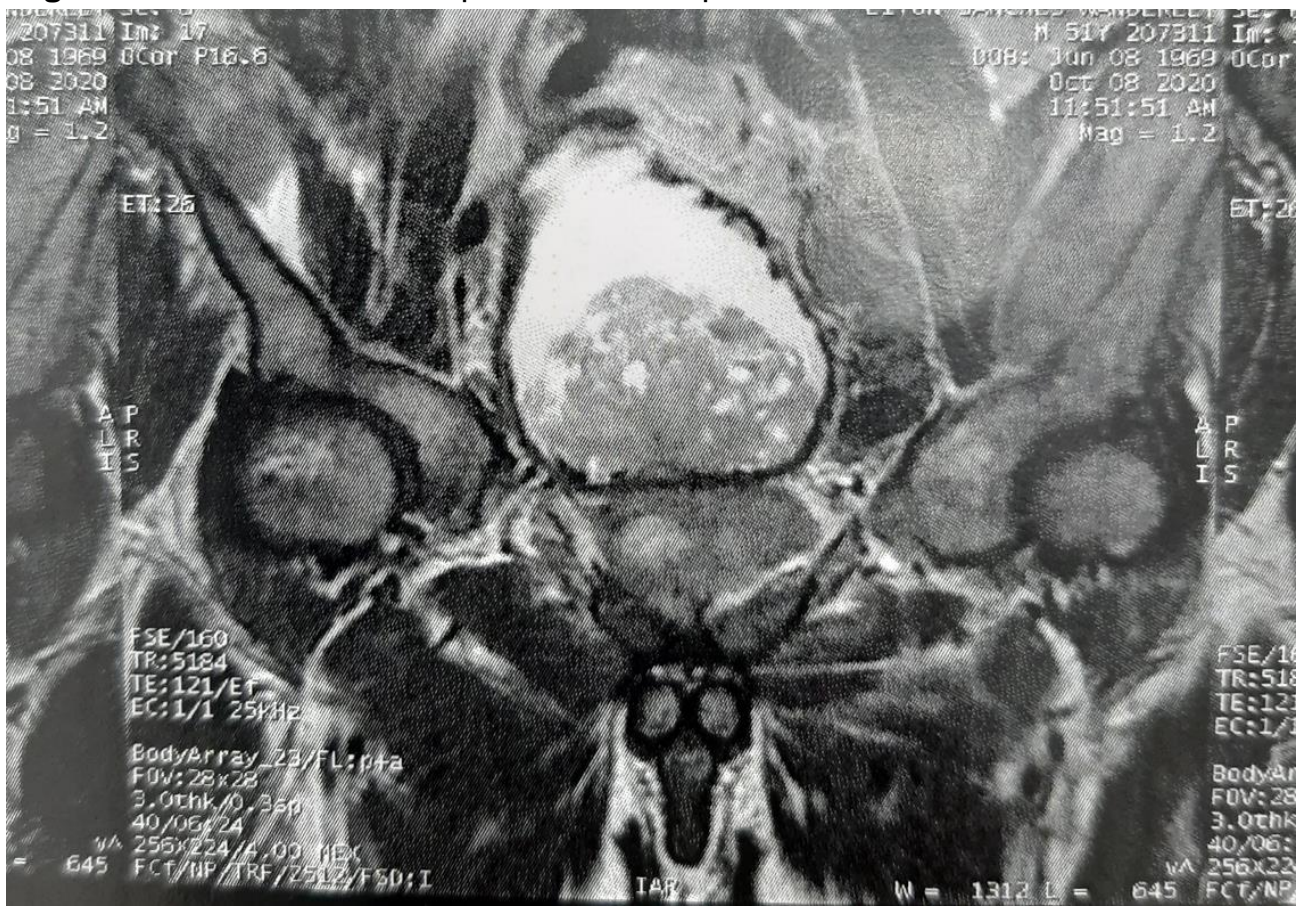

Fonte: Costa MS, et al., 2021.

Figura 4 - Ressonância Multiparamétrica da próstata em corte sagital.

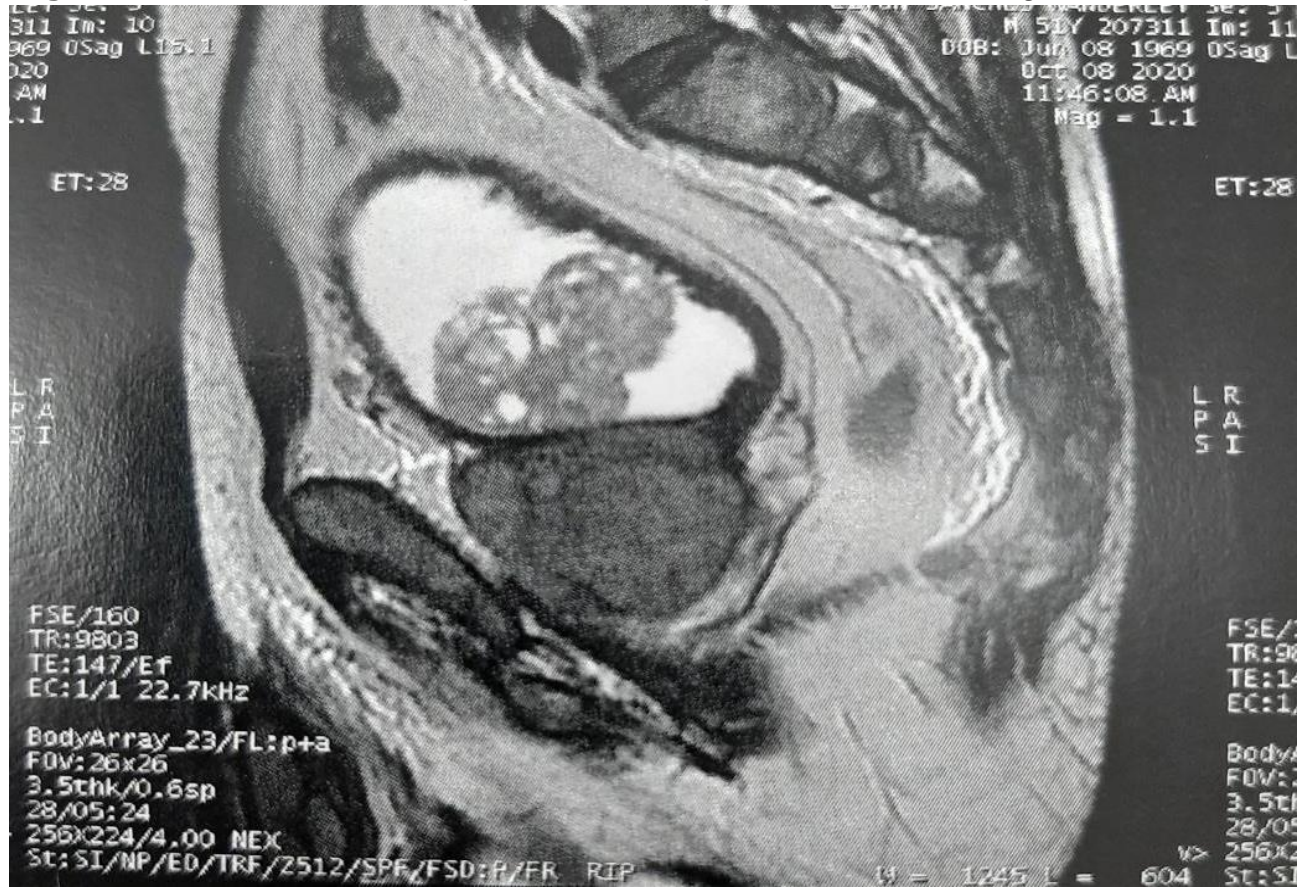

Fonte: Costa MS, et al., 2021.

A cintilografia óssea descreveu padrão cintilográfico compatível com doença óssea metastática disseminada mostrando áreas de aumento da concentração do radiotraçado em calota craniana, úmeros, rádio direito, escápulas, clavículas, esterno, arcos costais, coluna vertebral, ossos do quadril e fêmures (Figura 5).

O resultado histopatológico concluiu adenocarcinoma acinar usual em todos os 15 fragmentos analisados, com grau de Gleason 4+5=9. 
Figura 5 - Cintilografia óssea.

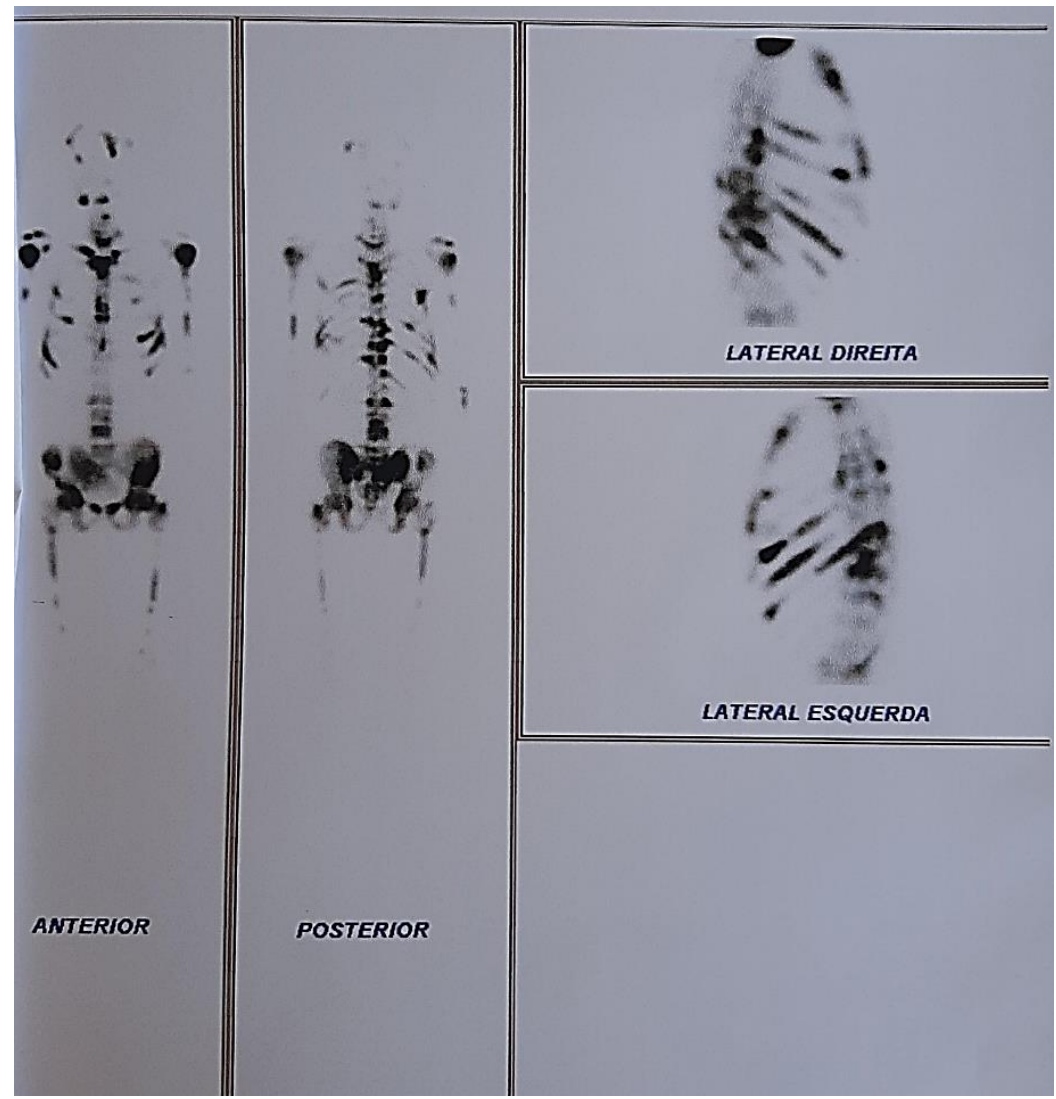

Fonte: Costa MS, et al., 2021.

Ressalta-se que o tempo entre a primeira consulta e o resultado da biópsia foi de aproximadamente 1 mês e 15 dias. Nesse período, foi estabelecido uma síndrome consumptiva com perda de peso maior que $10 \mathrm{~kg}$, além da necessidade de aumento de analgesia devido à dores osteoarticulares. Foram necessárias doses crescentes de Cloridrato de Tramadol endovenoso para controle da dor, inicialmente $200 \mathrm{mg} / \mathrm{dia}$ para $600 \mathrm{mg} / \mathrm{dia}$ e então progredindo para morfina endovenosa. Evoluiu com piora do estado geral, apresentando hematêmese e piora das dores ósseas e disúria.

Paciente necessitou de internação hospitalar para controle da dor e passagem de sonda vesical de demora na qual constatou a presença de hematúria na urina coletada. Durante a internação, manifestou episódios de hematêmese, sendo realizado Endoscopia Digestiva Alta (EDA) para esclarecimento do quadro de sangramento, constatando pangastrite edematosa leve. Os exames laboratoriais da internação apresentaram uma anemia significativa com hemoglobina de $6,4 \mathrm{~g} / \mathrm{dL}$, sendo necessário transfusão de 2 bolsas de hemoconcentrado.

Após controle do quadro hemorrágico, otimização da analgesia, o paciente recebeu alta com regular estado geral para realizar tratamento oncológico em um hospital do interior de São Paulo.

\section{DISCUSSÃO}

A incidência de câncer de próstata ajustado por idade aumentou na população dos EUA durante o final da década de 1980 e início da década de 1990 como resultado da triagem com PSA sérico. Desde então, a incidência diminuiu ou estabilizou na maioria das faixas etárias. Embora o carcinoma prostático de início precoce representa apenas uma pequena parte dos diagnósticos desta doença, a incidência neste grupo etário continua a aumentar. Esse fato se deve ao aumento da acessibilidade e sensibilidade analítica melhorada dos testes PSA atuais, o que tornou o diagnóstico em homens jovens mais comum (BANIAK N, et al., 2020). 
As neoplasias prostáticas de início precoce se diferem das neoplasias de idade avançada de variados modos. Primeiramente, homens com câncer prostático em estágio avançado, diagnosticados em idade jovem, apresentam mortalidade mais alta quando comparados aos homens idosos, excetuando indivíduos senis com mais de 80 anos. Tal fato sugere existência de diferenças biológicas entre estes tipos de neoplasias. Em segundo, o carcinoma de próstata de acometimento precoce apresenta forte componente genético, o que significa que pacientes acometidos por esse tipo de tumor maligno podem se beneficiar da avaliação do risco genético (BLEYER A, et al., 2019).

Por fim, embora a maioria destes pacientes sejam diagnosticados com doença de baixo risco, sua expectativa de vida prolongada os expõe à morbidade resultante dos efeitos deletérios e complicações do tratamento, que pode ter um impacto negativo na qualidade de vida dos jovens. E o longo prazo para progressão da doença, resultando em uma patologia de alta mortalidade. Embasados nisso, tem-se que as neoplasias prostáticas de início precoce apresentam um fenótipo distinto, tanto do ponto de vista etiológico quanto das manifestações e perspectivas clínicas, que merecem maior atenção (BLEYER A, et al., 2019).

Hoje, estima-se que a maioria dos indivíduos com diagnóstico de neoplasia prostática tenha um prognóstico favorável, com estimativas atuais para sobrevida relativa de 5 e 10 anos de 100,0\% e 98,7\%, respectivamente, resultados de pesquisas realizadas pelo Centro de Vigilância, Registros de Epidemiologia e Resultados Finais ("Surveillance, Epidemiology, and End Result" (SEER)), na população norte americana de homens diagnosticados com câncer de próstata durante 1994-2009 (SALINAS CA, et al., 2014).

Estes dados que refletem altas taxas de sobrevivência indicam que a maioria dos casos são identificados no início da história natural da doença, quando o tratamento com intenção curativa é possível e a enfermidade em estágio avançado representa apenas uma pequena proporção do total de diagnósticos. Ademais, os jovens com câncer podem ter menos comorbidades, as quais poderiam complicar a escolha do tratamento ou sua resposta imediata à terapêutica, além de serem mais propensos a receber tratamento agressivo do que homens idosos.

Entretanto, pacientes em estágios iniciais, suscetíveis ao tratamento curativo, não desenvolvem sinal ou sintoma relacionado à patologia. Como no caso amostrado, os sintomas surgem, em geral, na doença local avançada ou metastática. Fato alinhado à literatura, pois tendo em vista estudos publicados em 2020, Baniak $\mathrm{N}$, et al. (2020) concluíram que formas de metástase agressivas neoplásicas podem ser mais comuns em homens jovens.

Em estudo, publicado em 2009, Lin e outros analisaram dados SEER e descobriu que os homens diagnosticados com $\leq 55$ anos eram mais propensos a ter cânceres de baixo grau do que seus homólogos mais antigos. Especificamente, homens com diagnóstico de câncer estágio IV, aqueles com idade entre 3544 anos tiveram um risco 1,5 vezes maior de mortalidade específica por tumor de próstata do que homens com idade entre 65-74 anos. Resultados semelhantes, anteriormente descritos em estudo europeu, sugerem que o mau prognóstico observado em alguns homens com câncer de próstata de início precoce pode ser atribuído à doença severa, que esteja em estágio avançado ou alto grau no momento do diagnóstico ou ambos (BLEYER A, et al., 2019).

Embora o carcinoma prostático seja uma das principais neoplasias presentes na população masculina e, a incidência em jovens venha aumentando nas últimas décadas, ainda há baixa adesão ao rastreio. Segundo análise da teoria de gênero de Separavich MA e Canesqui AM (2013), essa evasão masculina se dá porque, durante a evolução humana, definiu-se uma cultura heteronormativa de que a prevenção e cuidados à saúde são tarefas socialmente idealizadas como femininas. Essas discrepâncias são relacionadas ao contexto de saúde versus enfermidade, deslegitimando a doença no homem e refletindo na sua baixa busca ao serviço de saúde.

Silva A, et al. (2020), em consonância, escalonou os fatores que impedem à população masculina ter acesso ao sistema de saúde. Dentre eles, os principais foram: 1) medo da enfermidade e do padecimento patológico assim como preocupação com o emprego e o bem-estar de seus dependentes; 2) vergonha e insegurança, por acreditarem que a doença afete sua masculinidade e a não aceitação das suas próprias 
condições biológicas; 3) receio da exposição corporal suscetível, questão enraizada ao machismo; e 4) Não reconhecimento da necessidade do cuidado à saúde inerente a masculinidade.

Considera-se também que, dentre os métodos de detecção da neoplasia prostática esteja o toque retal, um método invasivo que evidencia o desconforto e o preconceito culturalmente arraigados. Segundo Serra VS, et al. (2020), homens brasileiros ainda apresentam pudor extremo quanto a questões de cunho sexual. Ao exame, os entraves se manifestam em torno da perturbação da virilidade masculina, em especial pacientes idosos e de baixo nível socioeconômico educacional. Desta forma, tem-se como resultado a baixa aderência aos métodos de detecção de câncer prostático.

Embora, atualmente as diretrizes de prevenção e promoção de saúde estejam voltadas para o público a partir de 50 anos, devido sua maior incidência de doenças crônicas e degenerativas, o crescente acometimento do carcinoma prostático precoce permite discutir a necessidade de ampliar a abrangência etária das recomendações vigentes. Destaca-se a importância da implementação da análise das características clínicas e dinâmicas do PSA, que podem ser usadas no rastreio, graduação de risco e identificação de formas potencialmente agressivas da doença, em pacientes jovens (OLIVEIRA RAR, 2019).

Entretanto, apenas a medida de ampliação do público-alvo para rastreamento do câncer de próstata, não se faz suficiente para promoção de saúde. A conscientização da sociedade, sobre a importância dos cuidados constantes, e a educação em saúde voltados ao público masculino são fundamentais para o rompimento de crenças, preconceitos, medos e tabus retrógrados e danosos (MORAES MCL e SILVA MJ, 2017).

A literatura é deficitária em evidências científicas, para se estabelecer protocolos individualizados para abordagem do carcinoma prostático precoce. Este estudo apresenta um paciente sem histórico familiar ou de avaliação urológica preventiva prévia. Ademais, a neoplasia foi diagnosticada em estágio avançado, tornando o caso uma manifestação incomum e com baixa expectativa de sobrevida. Portanto, este estudo objetiva alarmar, profissionais de saúde e a população masculina, para valorização da ocorrência de neoplasia prostática em homem jovem e a importância da investigação de forma antecipada, proporcionando diagnóstico, tratamento e prognóstico promissor.

\section{REFERÊNCIAS}

1. ARAUJO, RM, et al. Adenocarcinoma prostático. Revista da Sociedade Brasileira de Clínica Médica, 2017; 15(3): 178-182.

2. BANIAK N, et al. Clinicopathologic and Molecular Characteristics of Prostate Cancer Diagnosed in Young Men Aged up to 45 Years. Histopathology, 2020.

3. BLEYER A, et al. Prostate cancer in young men: An emerging young adult and older adolescent challenge. Cancer, 2020; 126(1): 46-57.

4. CZORNY RCN, et al. Fatores de risco para o câncer de próstata: população de uma unidade básica de saúde. Cogitare Enfermagem, 2017; 22(4).

5. HUANG T-H, et al. Prostate cancer in young adults-Seventeen-year clinical experience of a single center. Journal of the Chinese Medical Association, 2017; 80(1): 39-43.

6. INCA. Câncer de Próstata. Instituto Nacional do Câncer, 2020. Disponivel em: https://www.inca.gov.br/tipos-decancer/cancer-de-prostata. Acessado em dezembro 2020.

7. LITWIN MS, TAN H-J. The Diagnosis and Treatment of Prostate Cancer. JAMA, 2017; 317(24).

8. MERRIEL SWD, et al. Prostate Cancer in Primary Care. Adv Ther. 2018; 35(9): 1285-1294.

9. MIKUZ G. Histologic classification of prostate cancer. Anal Quant Cytopathol Histpathol, 2015; 37(1):39-47.

10. MORAES MCL, et al. Uma questão masculina: conhecendo possíveis entraves para a realização dos exames de detecção do câncer de próstata. Revista Medica Herediana, 2017; 28(4): 230-235.

11. OLIVEIRA RAR. Análise da custo-efetividade do rastreamento e das modalidades terapêuticas do câncer de próstata. Tese (doutorado em Ciências Área de Concentração: Oncologia) - Fundação Antônio Prudente. São Paulo, 2019; $124 \mathrm{p}$.

12. QUIJADA PDS, et al. Câncer de próstata: retrato de uma realidade de pacientes em tratamento. Rev. enferm UFPE online, $2017 ; 11(6)$ : 2490-2499.

13. SALINAS CA, et al. Prostate cancer in young men: an important clinical entity. Nature Reviews Urology, 2014; 11(6).

14. SARRIS AB, et al. Câncer de próstata: uma breve revisão atualizada. Visão Acadêmica, 2018; 19(1)

15. SEPARAVICH MA, CANESQUI AM. Men's health and masculinities in the Brazilian Comprehensive Healthcare Policy for Men: a bibliographical review. Saúde e Sociedade, 2013; 22: 415-428. 
16. SERRA VS, et al. Saúde do homem: dificuldades vivenciadas a prevenção do câncer de próstata. Research, Society and Development, 2020; 9(11).

17. SHIH H-J, et al. Early-onset prostate cancer is associated with increased risks of disease progression and cancerspecific mortality. The Prostate, 2020; 81(2): 118-126.

18. SILVA A, et al. Saúde do homem: dificuldades encontradas pela população masculina para ter acesso aos serviços da unidade de saúde da família (USF). Brazilian Journal of Health Review, 2020; 3(2): 1966-1989.

19. VAZ DWN, et al. Retrato epidemiológico de pacientes internados com câncer de próstata em Belém-PA. Revista Brasileira de Educação e Saúde, 2020;10(2): 98-103. 